\title{
Single liver metastasis of a follicular variant of papillary thyroid carcinoma: a rare pattern of organ spread
}

\author{
Viviana Ostrovsky¹, Barak Bar-Zakai², Rafael Miller², Tal Schiller¹, Alena Kirzhner¹, \\ Hilla Knobler ${ }^{1}$ and Taiba Zornitzki1
}

1Diabetes, Endocrinology and Metabolic Disease Institute, Kaplan Medical Center, Hebrew University Medical School of Jerusalem, Rehovot, Israel and 2Surgery A Department, Kaplan Medical Center, Hebrew University Medical School of Jerusalem, Rehovot, Israel

\section{Correspondence} should be addressed to V Ostrovsky Email

viviana.ostrovsky@gmail.com

\section{Summary}

Well-differentiated thyroid cancer (WDTC), including papillary, follicular, and Hurthle-cell types, is characterized by a slow course and usually remain localized to the thyroid. However, a minority of these cases develop distant metastases with the most common sites being lungs, bones, and lymph nodes. Liver metastases of WDTC are rare and are usually found along with other distant metastases sites and in a multiple or diffuse pattern of spread. The recognition of distant metastasis in WDTC has a significant impact on the treatment and prognosis. However, because of their low incidence and awareness, distant metastases are often diagnosed late. Herein, we describe a case of a 71 years old woman who during routine surveillance of a follicular variant of papillary thyroid cancer (FV-PTC), 5 years after being treated for her primary thyroid tumor, was found to have a single liver metastasis and underwent liver segmental resection. This case highlights the importance of maintaining vigilant surveillance of patients with WDTC, and illustrates the possibility of unique metastasis at unexpected sites. Further studies are needed to understand the organ tropism of some WDTC leading to distant metastases development and to better prediction of an aggressive course.

\section{Learning points:}

- WDTC patients with distant metastases have a poor prognosis with a 10 -year survival of about $50 \%$. The most common sites of distant metastases are lung, bone and lymph nodes.

- Liver metastases are rare in WDTC, are usually multiple or diffuse and are found along with other distant metastases sites.

- Single liver metastasis of WDTC is an unexpected pattern of spread, and very few cases are reported in the literature.

- Rare sites of distant metastases in WDTC can manifest many years after the primary tumor, stressing the importance of maintaining vigilant surveillance.

- More studies are needed to predict which WDTC tumors may develop a more aggressive course, allowing clinicians to individualize patient management. 


\section{Background}

Papillary thyroid cancer (PTC) comprises about 85\% of well-differentiated thyroid cancer (WDTC) (1). The classic papillary (C-PTC) and follicular variant (FV-PTC) of PTC are the most common PTC subtypes, accounting for $55-65 \%$ and $23-41 \%$ of the cases, respectively (2). Historically, C-PTC and FV-PTC were believed to be low-risk with comparable treatment approach and prognosis. However, recently, The Cancer Genome Atlas (TCGA) research group demonstrated a clear difference in genetic profiles and clinical outcomes between these two subtypes and linked C-PTC to increased prevalence of thyroid capsule invasion, lymph node metastasis, disease recurrence and mortality (3).

Distant metastases in WDTC range from 4 to $15 \%$ and are the most frequent cause of WDTC-related death (4). The most common distant metastases sites in WDTC are lungs, followed by bone. Other rare distant metastases sites are brain, breast, liver, kidney, muscle and skin $(4,5)$.

Liver metastases are usually found along with other distant metastases sites, and nearly always are multiple or diffuse (5).

This study reports a rare case of a patient with FV-PTC who developed an unusual pattern of liver involvement, a single metastasis without evidence of other organ metastases.

\section{Case presentation}

A 65-year-old female underwent total thyroidectomy in December 2013 due to a $55 \mathrm{~mm}$ nodule in the left lobe of the thyroid gland. The histopathology findings showed a predominant FV-PTC with a 10\% component of insular type. In addition, there were foci of solid pattern, massive invasion and reduplication of the capsule and several foci of vascular invasion. Extra thyroidal extension was not identified. A $3 \mathrm{~mm}$ micropapillary carcinoma was seen in the right lobe.

Her past medical history included nodular goiter for which she was followed in an endocrinology clinic, dyslipidemia and hypertension. There was no history of exposure to head or neck radiation. Her family history included two siblings diagnosed with PTC; a sister who underwent total thyroidectomy at the age of 62 and a brother who underwent right hemithyroidectomy at the age of 71 due to PTC. There was no available information regarding her parents. At the time of the surgery, thyroid function tests were normal, and serum thyroglobulin (Tg) was remarkably high, $4013 \mathrm{ng} / \mathrm{mL}$ (reference value $0.8-55 \mathrm{ng} / \mathrm{mL})$.

\section{Investigation}

Postoperatively, she received $100 \mathrm{mCi}$ 131-radioiodine (I-131). A following whole-body scan (WBS) with SPECT/CT showed small foci of iodine accumulation

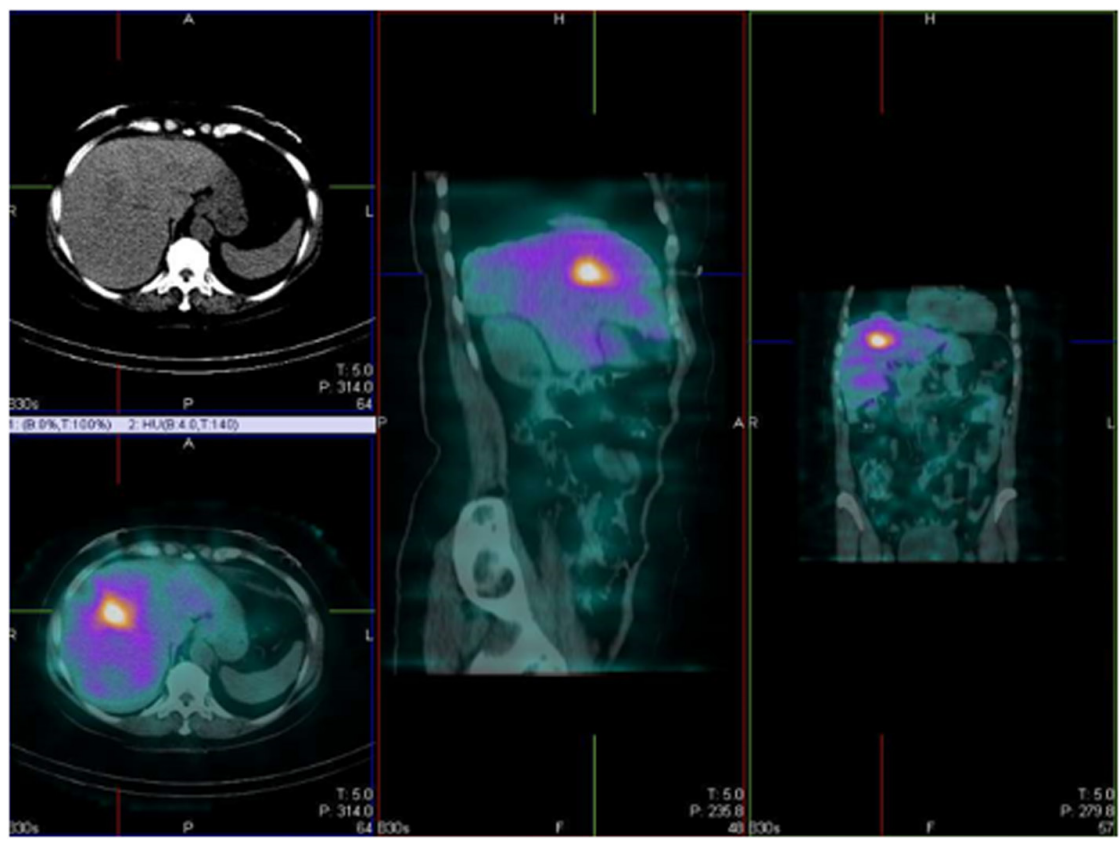

Figure 1

WBS/SPECT CT showed a solitary radioiodine-avid mass in the right segment of the liver. 
at the thyroid bed compatible with remnant thyroid tissue. At this time, thyroid-stimulating hormone (TSH) was $95.5 \mathrm{IU} / \mathrm{L}$ (reference value $0.50-4.78$ ), $\mathrm{Tg}$ decreased to $77 \mathrm{ng} / \mathrm{mL}$ and thyroglobulin antibodies (Tg- $\mathrm{Ab}$ ) were negative. Six months later, a neck ultrasound (US) did not demonstrate any findings and a thyrogen test performed a year post-surgery revealed a $\mathrm{Tg}$ of $1.4 \mathrm{ng} / \mathrm{mL}$ while TSH was $41 \mathrm{IU} / \mathrm{L}$.

According to the Joint Committee on Cancer (AJCC 8th Edition) and the TNM classification system (6), all the above were consistent with STAGE II-T3a, NX, M0 and with an intermediate risk of recurrence.

\section{Outcome and follow-up}

At follow-up visits, she was well controlled with L-T4 therapy. Tg levels remained undetectable until 2018 when a gradual rise was noted, despite suppressed TSH. Repeated cervical US and a positron emission CT scan (PET-CT) showed no abnormal findings.

The Tg levels continued to rise, up to $118 \mathrm{ng} / \mathrm{mL}$, and the second dose of I-131 (170 mCi) was administered. A WBS/SPECT-CT scan 9 days following treatment revealed a solitary radioiodine-avid mass in the right segment of the liver (segment 8) (Fig. 1). To confirm this finding and to assess the possibility of other foci of metastatic tumors obscured by the intense liver lesion, a repeated PET-CT scan was conducted and confirmed the findings of a solitary liver lesion without any other abnormalities. Despite the second I-131 therapy, Tg levels continued to increase.

An abdominal contrast-enhanced CT scan that was done to further define the finding, revealed a 2 $\mathrm{cm}$ hypodense lesion in segment 8 of the liver (Fig. 2A). During February 2020, the patient underwent an uncomplicated resection of the isolated liver metastasis. The intraoperative and post-resection macroscopy of the metastasis are shown in Fig. 2B and C.

The microscopic features of the metastatic lesion showed the same histopathology as the primary thyroid tumor consistent with FV-PTC (Fig. 3A, B, C and D). There was no evidence of insular type. Immunohistochemical analysis showed positive staining for TTF-1 and Tg (Fig. 3C and D) confirming the lesion's thyroidal origin. Surgical margins were free of tumor involvement. Molecular analysis of the tumor did not reveal $B R A F^{V}{ }^{V 00 E}$ mutation and the index Ki67 was less than $2 \%$. Three months following surgery $\mathrm{Tg}$ levels decreased to $1.75 \mathrm{ng} / \mathrm{mL}$.

\section{Discussion}

We describe a case of an elderly woman who underwent a PTC resection in 2013. There were two unfavorable initial characteristics: age above 55 years and a large tumor at presentation. However, the tumor histology was compatible with FV-PTC and there was no evidence of distant metastasis. Moreover, during a relatively long follow-up period, the unstimulated Tg levels were undetectable. Only, 5 years later, due to a gradual increase in $\mathrm{Tg}$ levels, a detailed evaluation was conducted that revealed a solitary liver metastasis. The patient underwent a resection confirming that it was a single metastasis of the primary FV-PTC.

WDTC is characterized by a slow and indolent course, with an excellent 10-year survival rate of 80-95\%. However, WDTC patients with distant metastases have a poor prognosis with a 10 -year survival of about $50 \%(4,7)$.
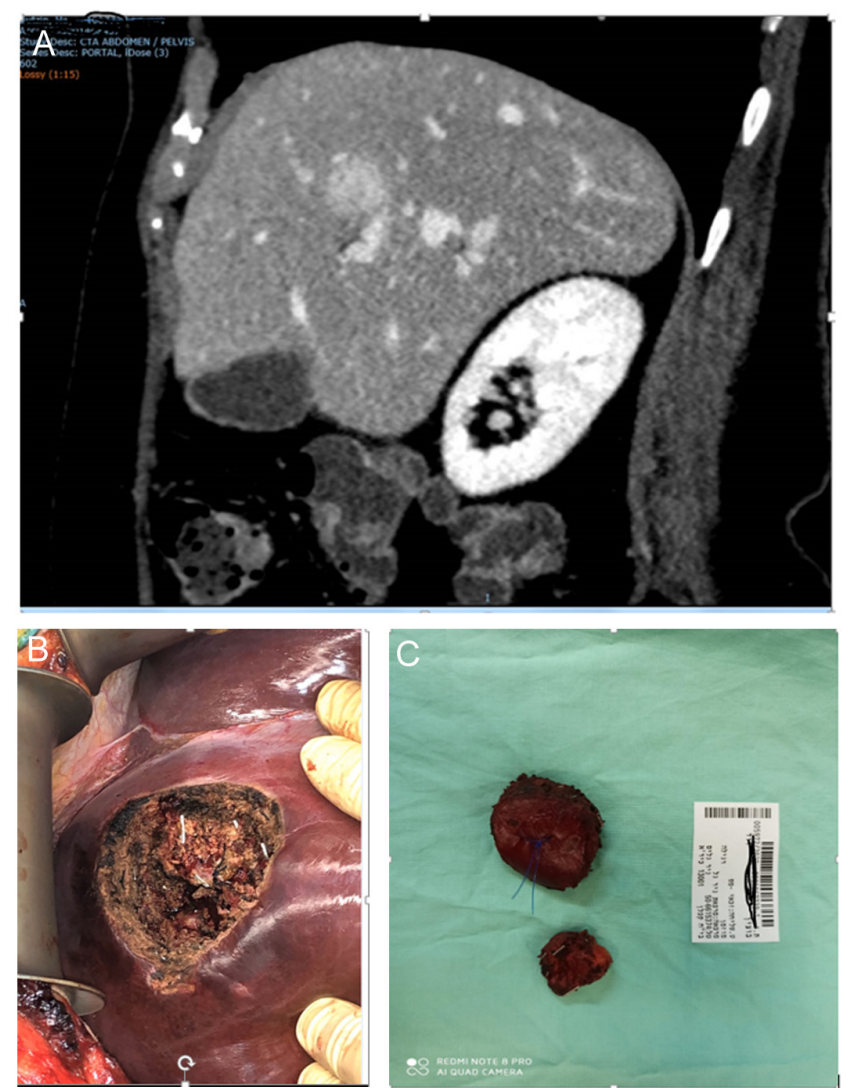

\section{Figure 2}

(A) Coronal contrast-enhanced CT of the abdomen shows a hypodense lesion $2 \mathrm{~cm}$ in diameter in the segment 8 of the liver. The lesion undergoes strong enhancement in the late arterial phase of the scan, and remains enhanced in the portal venous phase of the scan. The lesion is situated between the middle hepatic vein, which is above the lesion, and the posterior branch of the right portal vein. (B) The gross hepatic metastasis seen intraoperatively. 

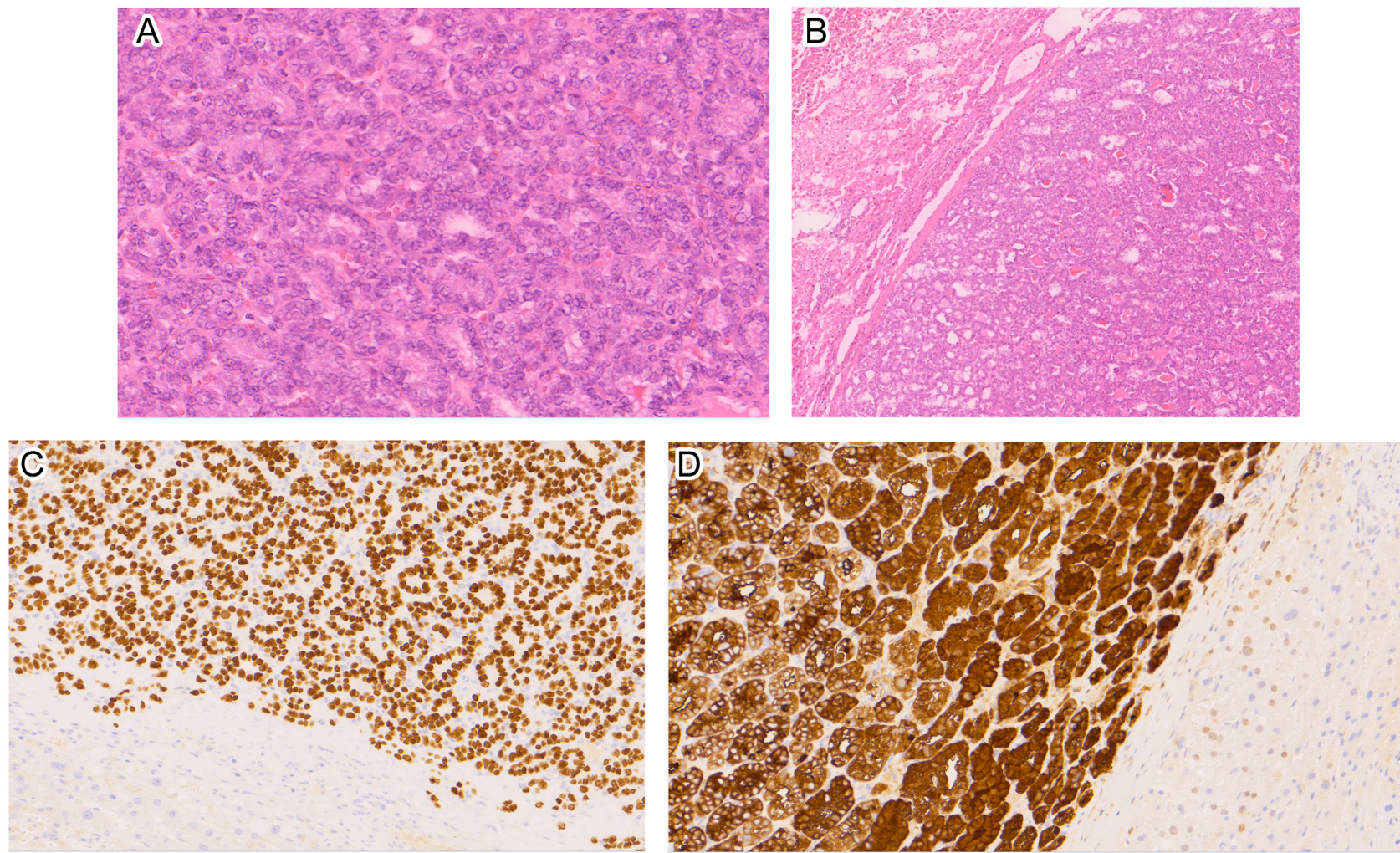

Figure 3

(A) Hematoxylin-eosin stain (H\&E), $\times 200$ magnification. The tumor is characterized by follicular growth pattern with colloid, composed of cells that show enlarged nuclei with overlapping, nuclear grooves and pseudoinclusions. The features are compatible with FV-PTC. (B) H\&E, $\times 100$ magnification. Metastatic papillary thyroid carcinoma. Liver tissue in the left. (C) $\times 200$ magnification, TTF-1 immunohistochemical stain. Strong nuclear staining in carcinoma cells. (D) $\times 200$ magnification, thyroglobulin immunohistochemical stain. Positive stain highlighted follicular growth pattern.

The liver is a rare site of distant metastases in WDTC with a reported frequency of only $0.5 \%$ (7). A retrospective study of a single center that evaluated patients with WDTC and distal metastases between 1970 and 2014, found liver metastases in only 5 out of 138 patients. Four of these patients also had lung metastases, two also had bone metastases, and one also had brain metastases. These results accord with previous studies showing that liver metastases in WDTC are usually found with evidence of other distant metastases sites, and nearly always are multiple or diffuse.

Madani et al. proposed several underlying mechanisms for the organ tropism and the differences in the biological behavior of thyroid cancer subtypes. Such mechanisms include involvement of a variety of signaling pathways, such as MAPK and PI3K-AKT, tumor immunology, molecular mediators of organ tropism and the theory of genetic heterogeneity within a primary tumor due to the presence of a subpopulation of cancer stem cells (7). However, all of these proposed mechanisms still fall short of explaining the phenomena of rare organ metastatic lesions. In addition, as demonstrated by our case, rare metastases of thyroid cancer can manifest years after discovering the primary tumor $(7,8)$. Therefore, there is a need for longer surveillances of WDTC patients (9).

Disease-specific mortality and the risk of recurrence is higher in patients with BRAF mutation and BRAFV600E mutation is tightly linked to aggressive clinicalpathological features (1). However, interestingly in our case, the molecular analysis did not reveal BRAF mutation and the Ki-67 index was low.

Our patient had a family history of two first-degree relatives with PTC. Familial non-medullary thyroid cancer (FNMTC) may be a component of familial cancer syndromes (Gardner and Cowden syndrome, Carney complex type 1, Werner and DICER1 syndromes), but the majority of FNMTC are non-syndromic. A prospective screening study of kindred with FNMTC reported a high rate of thyroid cancer in families with three or more firstdegree relatives affected (10).

At least ten similar cases were reported in the literature (4). Three cases were male and seven were female. The average age was 63 years old (range 32-85). The histopathology of the primary tumor was papillary 
in four, follicular in five patients and Hurtle cell in one patient. Our case shows similar clinical and pathological characteristics to the others published.

The patient underwent resection of the solitary liver metastasis that did not respond to a repeated I-131 treatment. Although I-131 is currently the treatment of choice for I-131 avid metastases (1), surgical resection offers more radical treatment and should be considered, especially for a solitary metastasis (4).

In conclusion, WDTC patients have an excellent prognosis with a life expectancy not significantly different from the general population after therapy. Nevertheless, accurate surveillance for possible recurrence is recommended for long-term follow-up. This case highlights the possibility of a unique metastasis at an unexpected site.

\section{Declaration of interest}

The authors declare that there is no conflict of interest that could be perceived as prejudicing the impartiality of the research reported.

\section{Funding}

This work did not receive any specific grant from any funding agency in the public, commercial, or not-for-profit sector.

\section{Author contribution statement}

H Knobler and T Zornitzki are co-senior authors.

\section{Acknowledgements}

The authors would like to thank Oleg Sukmanov MD for pathology work and Shahar Ish-Shalom PHD for molecular analysis contribution.

\section{References}

1 Haugen BR, Alexander EK, Bible KC, Doherty GM, Mandel SJ, Nikiforov YE, Pacini F, Randolph GW, Sawka AM, Schlumberger, et al. 2015 American Thyroid Association management guidelines for adult patients with thyroid nodules and differentiated thyroid cancer. Thyroid 201626 1-133. (https://doi.org/10.1089/ thy.2015.0020)

2 Henke LE, Pfeifer JD, Baranski TJ, DeWees T \& Grigsby PW. Longterm outcomes of follicular variant vs classic papillary thyroid carcinoma. Endocrine Connections 20187 1226-1235. (https://doi. org/10.1530/EC-18-0264)

3 Cancer Genome Atlas Research Network. Integrated genomic characterization of papillary thyroid carcinoma. Cell 2014159 676-690. (https://doi.org/10.1016/j.cell.2014.09.050)

4 Song HJ, Xue YL, Xu YH, Qiu ZL \& Lug QY. Rare metastases of differentiated thyroid carcinoma: pictorial review. EndocrineRelated Cancer 201118 R165-R174. (https://doi.org/10.1530/ERC11-0068)

5 Hirsch D, Levy S, Tsvetov G, Gorshtein A, Slutzky-Shraga I, Akirov A, Robenshtok E, Shimon I \& Benbassat CA. Long-term outcomes and prognostic factors in patients with differentiated thyroid cancer and distant metastases. Endocrine Practice 201723 1193-1200. (https:// doi.org/10.4158/EP171924.OR)

6 Schmidbauer B, Menhart K, Hellwig D \& Grosse J. Differentiated thyroid cancer - treatment: state of the art. International Journal of Molecular Sciences 201718 181-177. (https://doi.org/10.3390/ ijms18061292)

7 Madani A, Jozaghi Y, Tabah R, How J \& Mitmaker E. Rare metastases of well-differentiated thyroid cancers: a systematic review. Annals of Surgical Oncology 201522 460-466. (https://doi.org/10.1245/s10434014-4058-y)

8 Passler C, Prager G, Scheuba C, Niederle BE, Kaserer K, Zettinig G \& Niederle B. Follicular variant of papillary thyroid carcinoma: a long term follow-up. Archives of Surgery 2003138 1362-1366. (https://doi. org/10.1001/archsurg.138.12.1362)

9 Song HJ, Xue YL, Qiu ZL \& Luo QY. Uncommon metastases from differentiated thyroid carcinoma. Hellenic Journal of Nuclear Medicine 201215 233-240. (https://doi.org/10.1967/s002449910059)

10 Klubo-Gwiezdzinska J, Yang L, Merkel R, Patel D, Nilubol N, Merino MJ, Skarulis M, Sadowski SM \& Kebebew E. Results of screening in familial non-medullary thyroid cancer. Thyroid 201727 1017-1024. (https://doi.org/10.1089/thy.2016.0668)

Received in final form 17 June 2020 Accepted 29 July 2020 